\title{
A Uniform Approach to Fundamental Sequences and Hierarchies
}

\author{
Wilfried Buchholz $^{1}$, Adam Cichon ${ }^{2}$ and Andreas Weiermann ${ }^{3}$ \\ ${ }^{1}$ Mathematisches Institut der Universität München, \\ Theresienstraße 39, D-80333 München, Germany ${ }^{1)}$ \\ ${ }^{2}$ INRIA-Lorraine-CNRS-CRIN, BP 101, F-54602 Villers-lès-Nancy, France ${ }^{2)}$ \\ ${ }^{3}$ Institut für mathematische Logik und Grundlagenforschung \\ der Westfälischen Wilhelms-Universität Münster, \\ Einsteinstraße 62, D-48149 Münster, Germany3)
}

\begin{abstract}
In this article we give a unifying approach to the theory of fundamental sequences and their related Hardy hierarchies of number-theoretic functions and we show the equivalence of the new approach with the classical one.
\end{abstract}

Mathematics Subject Classification: 03D20, 03F15, 03E10.

Keywords: Natural well-orderings, Subrecursive hierarchies, Hardy hierarchies, Fundamental sequences, Descent functions.

\section{Introduction}

A fascinating result of (Gentzen-style) proof theory is the characterization of the provably total functions of Peano-arithmetic in terms of KREISEL's ordinal recursive functions (see KREISEL [9]), or alternatively, in terms of the $\prec$-descent recursive functions (see Smith [13], Takeuti [15] or Friedman-Sheard [7]), where $\prec$ denotes a standard representation of $\varepsilon_{0}$ in the natural numbers. This class of functions can also be characterized by hierarchies of number-theoretic functions which are defined relative to the system of standard fundamental sequences for the ordinals less than $\varepsilon_{0}$. Examples are here the Hardy hierarchy, the extended Grzegorczyk hierarchy and a hierarchy which is based on iterated enumeration (SCHWICHTENBERG [14], WAINER [17]). A generalization of the latter concepts would still seem to be problematic. There were some results concerning $\Gamma_{0}$, the proof-theoretic ordinal of predicative analysis, or $\eta_{\nu}$, the proof-theoretic ordinal of $I D_{\nu}$ (cf. ZEMKE [22], BUCHHOLz [2]). But the larger the countable ordinal in question, the harder becomes the problem of assigning an appropriate (Bachmann) system of fundamental sequences. In his article [5] CicHON proposes implicitly a very simple and general method for approaching

\footnotetext{
1) e-mail: buchholz@rz.mathematik.uni-muenchen.de

2) e-mail: cichon@loria.crin.fr

3)e-mail: weierma@math.uni-muenster.de
} 
this problem. His approach is based on the interplay between Bachmann systems of fundamental sequences and a term-complexity-function (which we call norm from now on). The importance of a norm function is already implicit in the literature (see Zемке [22] and Sмітн [13]). The new idea is to define a Bachmann system of fundamental sequences $\left(\alpha[n]_{C}\right)_{n \in \mathbb{N}}$ in terms of the norm instead of defining it by refering to some normal form representation of the respective ordinals. For example, if $N: \varepsilon_{0} \longrightarrow \mathbb{N}$ is a norm function such that $N 0=0, N(\alpha+1) \leq N \alpha+1$ for all $\alpha<\varepsilon_{0}$ and $\operatorname{card}\{\beta<\alpha: N \beta<d\}$ is finite for all $d \in \mathbb{N}$ and $\alpha<\varepsilon_{0}$, then we put (see Cichon [5]):

$$
\alpha[n]_{C}:=\max \{\beta<\alpha: N \beta \leq N \alpha+n\}{ }^{4)}
$$

Moreover it turns out that the Hardy hierarchies can be defined in terms of the norm without any reference to fundamental sequences at all. If we put

$$
H_{\alpha}(n):=\max \left\{\{n\} \cup\left\{H_{\beta}(n+1): \beta<\alpha \& N \beta \leq N \alpha+n\right\}\right\},
$$

then this hierarchy coincides with (a slight variant of) the usual Hardy hierarchy with respect to $\cdot[\cdot]_{C}$, namely for $\alpha>0$ one has $H_{\alpha}(n)=H_{\alpha[n]_{C}}(n+1)$.

An immediate consequence of this definition which is very useful for applications is the following effective majorization property:

$$
\alpha<\beta \& N \alpha \leq N \beta+n \Rightarrow H_{\alpha}(n)<H_{\beta}(n) .
$$

Of course (EMP) yields the usual majorization property of the Hardy hierarchy, namely

$$
\alpha<\beta \Rightarrow(\exists m)(\forall n \geq m)\left[H_{\alpha}(n)<H_{\beta}(n)\right] .
$$

But, in additition, (EMP) gives an useful effective criterion how to compute a natural number $m$ such that $H_{\alpha}(n)<H_{\beta}(n)$ holds for all $n \geq m$. Simply pick $m$ such that $N \alpha \leq N \beta+m$. In the more traditional approach the verification of assertions like $(\mathrm{EMP})$ (especially for proof-theoretic ordinals larger than $\varepsilon_{0}$ ) is not always immediate (see ZEMKE [22]).

In this article we investigate the consequences of this new approach to the theory of Hardy hierarchies and we will compare this approach with the usual one. It turns out that under some natural assumptions the new approach is equivalent to the old one. The new approach has proven useful in WEIERMANN [19] where a comparatively simple and straightforward characterization of the provably total functions of Peanoarithmetic is given in terms of a Cichon-style Hardy hierarchy. ${ }^{5}$ )

In Section 1 we develop a general theory of normed Bachmann systems and their related Hardy hierarchies. In Section 2, following WAINER [16], we compare the Hardy hierarchies with certain hierarchies of primitive recursively norm bounded descent functions. This also provides means for comparing Hardy hierarchies belonging to different normed Bachmann systems. In Section 3 we relate Cichon's approach to the more traditional theory of normed Bachmann systems presented in Section 1. In

\footnotetext{
4) A similar (but not equivalent) definition is contained in FrIEDMAN-SHEARD [7, Lemma 1.31]

5) The new approach has recently also been proved useful for bounding derivation lengths of rewrite systems with slow growing functions (see WEIERMANN [20]) and for investigations on slow versus fast growing for proof-theoretic ordinals larger than the first subrecursive ordinal (see WEIERMANN [21]).
} 
Section 4 we give some applications to $\varepsilon_{0}$ and other proof-theoretic ordinals. Moreover we reformulate the main result of Section 2 in a way which avoids any reference to fundamental sequences.

\section{Fundamental sequences and Hardy hierarchies}

Let $\omega$ be the least infinite ordinal and let $\tau$ be fixed such that $\left(\exists \tau_{0}>0\right)\left[\tau=\omega^{\omega} \cdot \tau_{0}\right]$. In the following $\alpha, \beta, \gamma, \xi$ range over ordinals less than $\tau$, and $i, j, k, l, m, n$ over natural numbers (finite ordinals). The set of natural numbers is denoted by $\mathbb{N}$ and the set of limit ordinal less than $\tau$ is denoted by Lim.

Definition. Let $\cdot[\cdot]: \tau \times \mathbb{N} \longrightarrow \tau$ and $N: \tau \longrightarrow \mathbb{N}$.

$(1) \cdot[\cdot]$ is called a system of fundamental sequences or an assignment of fundamental sequences if

(B1) $(\forall \alpha, n)[0[n]=0 \&(\alpha+1)[n]=\alpha \&[\alpha \in \operatorname{Lim} \Rightarrow \alpha[n]<\alpha[n+1]<\alpha]]$.

(2) The Hardy hierarchy $\left(H_{\alpha}\right)_{\alpha<\tau}$ for $\langle\tau, \cdot[\cdot]\rangle$ is defined by

$$
H_{0}(n):=n, \text { and } H_{\alpha}(n):=H_{\alpha[n]}(n+1) \text { for } \alpha>0 .
$$

Now let $\cdot[\cdot]$ be an assignment of fundamental sequences.

(3) $\langle\tau, \cdot[\cdot]\rangle$ is called a Bachmann system if

(B2) $(\forall \alpha, \beta, n)[\alpha[n]<\beta<\alpha \Rightarrow \alpha[n] \leq \beta[0]]$.

(4) We say that $\cdot[\cdot]$ is compatible with $N$ and call $\langle\tau, \cdot[\cdot], N\rangle$ a normed Bachmann system if

(B4) $(\forall \alpha \in \operatorname{Lim})[N \alpha \leq N \alpha[0]+1]$.

(5) We call $\langle\tau, \cdot[\cdot], N\rangle$ a regular Bachmann system if $(\forall \beta<\alpha)[\beta \leq \alpha[N \beta]]$.

(6) $N$ is called a norm on $\tau$ if $(\forall \alpha, n)[\operatorname{card}\{\beta<\alpha: N \beta \leq n\}<\omega]$.

Remarks. In Lemma 3 we shall prove that $\sup \{\alpha[n]: n \in \mathbb{N}\}=\alpha$ for Bachmann systems and limit ordinals $\alpha$. Since we shall concentrate mainly on Bachmann systems in this article we do not demand this additional property in (B1). Our definition of the Hardy hierarchy is a slight modification of the usual Hardy hierarchy which is defined by $H_{0}(n):=n, H_{\alpha+1}(n):=H_{\alpha}(n+1)$, and $H_{\alpha}(n):=H_{\alpha[n]}(n)$ for limit ordinals $\alpha$. Our choice has some technical advantages but our approach can be carried out in the same way also for the classical Hardy hierachy. Condition (B2) is the socalled Bachmann property (see Schmid [12]). The usefulness of this condition in investigations on subrecursive hierarchies is discussed for example in Rose [11]. The idea of considering normed systems of fundamental sequences is already contained in ZEMKE [22]. Compared with his definition our approach is more restrictive (see Lemma 1 below). The concept of a norm is contained in SMITH [13]. A slightly different concept of Bachmann system, namely the concept of a structured tree ordinal has been investigated in WAINER [18].

Le m m a 1. If $\langle\tau, \cdot[\cdot], N\rangle$ is a normed Bachmann system, then

(a) $\langle\tau, \cdot[\cdot]\rangle$ is a Bachmann system,

(b) $N$ is a norm on $\tau$,

(c) $\langle\tau, \cdot[\cdot], N\rangle$ is a regular Bachmann system. 
Proof.

(a). Let $\alpha[n]<\beta<\alpha$ and assume $\beta[0]<\alpha[n]$. Then by (B1), (B3), (B4) we get $N \alpha[n]<N \beta \leq N \beta[0]+1 \leq N \alpha[n]$. Contradiction.

(b). This follows by induction on $\alpha$ from

$$
\{\beta<\alpha: N \beta \leq n\} \subseteq\{\beta \leq \alpha[n]: N \beta \leq n\} .
$$

For $\alpha \notin \operatorname{Lim}(*)$ is trivial; if $\alpha \in \operatorname{Lim}$, then by (B3) we have $(\forall n)[N \alpha[n]<N \alpha[n+1]]$, and therefore $n \leq N \alpha[n]$; (B3) also yields $(\forall \beta<\alpha)[N \beta \leq N \alpha[n] \Rightarrow \beta \leq \alpha[n]]$.

(c). Let $\alpha \in \operatorname{Lim}$. Then by (B3) we have $(\forall n)[n \leq N \alpha[n]]$, so, in particular, $N \beta \leq N \alpha[N \beta]$. The latter together with (B3) yields $[\beta<\alpha \Rightarrow \beta \leq \alpha[N \beta]]$.

$\mathrm{Rem}$ a $\mathrm{k}$. Lemma $1(\mathrm{c})$ yields that normed Bachmann systems are regulated in the sense of ZEMKE [22]. It can be shown that the conclusion of Lemma 1(c) can be sharpened to $(\forall \beta<\alpha)[\beta \leq \alpha[N \beta-(N \alpha-1)]]$.

In the following, we write $\alpha[0]^{i}$ to denote $\alpha \underbrace{[0] \ldots[0]}_{i \text {-times }}$.

Le m ma 2. Let $\langle\tau, \cdot[\cdot]\rangle$ be a system of fundamental sequences and let $G: \tau \longrightarrow \mathbb{N}$ be defined by $G \alpha:=\min \left\{i: \alpha[0]^{i}=0\right\}$. Then we have:

(a) If $\langle\tau, \cdot[\cdot]\rangle$ is a Bachmann system, then $\langle\tau, \cdot[\cdot], G\rangle$ is a normed Bachmann system and $(\forall \alpha)[\{\beta: \beta[0] \leq \alpha<\beta\}<\omega]$.

(b) If $(\forall \alpha)[\{\beta: \beta[0]=\alpha\}<\omega]$, then $G$ is a norm.

Proof.

(a). If $\alpha[n]<\beta<\alpha$, then $\alpha[n]=\beta[0]^{i}$ for some $i>0$, and thus $G \alpha[n]<G \beta$. Obviously $G \alpha=G \alpha[0]+1$ for each $\alpha>0$. Thus $\langle\tau, \cdot[\cdot], G\rangle$ is a normed Bachmann system. By Lemma 1 (b) $G$ is a norm and therefore $(\forall \alpha)[\{\beta: G \beta \leq G \alpha+1\}<\omega]$. Since $\langle\tau, \cdot[\cdot], G\rangle$ is a normed Bachmann system, we also have $\{\beta: \beta[0] \leq \alpha<\beta\} \subseteq$ $\{\beta: G \beta \leq G \alpha+1\}$.

(b). Assume that there are $\alpha$ and $d<\omega$ such that $\{\beta<\alpha: G \beta<d\}$ is infinite. Then $X:=\{\beta<\alpha: G \beta=k\}$ is infinite for some minimal $k<d$. Then $k>0$, since $\beta[0]^{0}=\beta>0$, if $\beta>0$. Put $Y:=\{\beta[0]: \beta \in X\}$. The minimality of $k$ yields that $Y$ is finite. So there is a $\gamma$ such that $\gamma=\beta[0]$ for infinitely many $\beta \in X$. This contradicts the assumption.

For the remainder of this section let $\cdot[\cdot]: \tau \times \mathbb{N} \longrightarrow \tau$ be fixed such that $\langle\tau, \cdot[\cdot], N\rangle$ is a regular Bachmann system, and let $\left(H_{\alpha}\right)_{\alpha<\tau}$ be the Hardy hierarchy for $\langle\tau, \cdot[\cdot]\rangle$.

Le m m a 3 .

(a) $H_{\alpha}(n)<H_{\alpha}(n+1)$.

(b) $\beta[m]<\alpha<\beta \Rightarrow H_{\beta[m]}(n)<H_{\alpha}(n)$.

(c) $0<\alpha \& m \leq n \Rightarrow H_{\alpha[m]}(n+1) \leq H_{\alpha}(n)$.

(d) $(\forall \beta<\alpha)\left[N \beta \leq n \Rightarrow H_{\beta}(n+1) \leq H_{\alpha}(n)\right]$.

(e) $\alpha \in \operatorname{Lim} \Rightarrow \alpha=\sup \{\alpha[n]: n \in \mathbb{N}\}$.

(f) $H_{\alpha}(n)=\min \{k \geq n: \alpha[n][n+1] \ldots[k-1]=0\}=\min \{k: \alpha[n: k]=0\}$, with $\alpha[n: k]:=\alpha+(n-k)$, for $k \leq n$, and $\alpha[n: k+1]:=(\alpha[n: k])[k]$, for $k \geq n$. 
Proof (compare the proof of Cichon [3, Theorem 2]).

(a) and (b) are proved simultaneously by induction on $\alpha$ : Let $\alpha>0$. Then

$$
H_{\alpha}(n)=H_{\alpha[n]}(n+1)<H_{\alpha[n]}(n+2)<H_{\alpha[n+1]}(n+2)=H_{\alpha}(n+1),
$$

and if $\beta[m]<\alpha<\beta$, then $\beta[m] \leq \alpha[n]<\beta$ and

$$
H_{\beta[m]}(n) \leq H_{\alpha[n]}(n)<H_{\alpha[n]}(n+1) .
$$

(c) follows from (b).

(d) is proved by induction on $\alpha$ : Let $\beta<\alpha$ and $N \beta \leq n$. Then $\beta \leq \alpha[N \beta] \leq \alpha[n]$. If $\beta=\alpha[n]$, then $H_{\beta}(n+1)=H_{\alpha}(n)$. If $\beta<\alpha[n]$, then [by I.H. and (a)] $H_{\beta}(n+1)<$ $H_{\beta}(n+2) \leq H_{\alpha[n]}(n+1)=H_{\alpha}(n)$.

(e). Suppose $\beta<\alpha$ and $(\forall m)[\alpha[m]<\beta]$. Then by (b) we have

$$
(\forall m)\left[H_{\alpha[m]}(0)<H_{\alpha[m+1]}(0)<H_{\beta}(0)\right] .
$$

Contradiction.

(f). Let $m:=\min \{k: \alpha[n: k]=0\}$. Then $m \geq n$ and, by definition,

$$
H_{\alpha[n: i]}(i)=H_{\alpha[n: i+1]}(i+1), \text { for } n \leq i<m .
$$

Hence $H_{\alpha}(n)=H_{\alpha[n: n]}(n)=H_{\alpha[n: m]}(m)=H_{0}(m)=m$.

Definition.

$$
\begin{aligned}
& N F(\alpha, \beta): \Leftrightarrow \alpha, \beta>0 \\
& \quad \&\left(\exists \alpha_{0}, \ldots, \alpha_{m}, \beta_{0}, \ldots, \beta_{n}\right)\left[\alpha=\omega^{\alpha_{0}}+\ldots+\omega^{\alpha_{m}}\right. \\
& \left.\quad \& \beta=\omega^{\beta_{0}}+\ldots+\omega^{\beta_{n}} \& \alpha_{0} \geq \ldots \geq \alpha_{m} \geq \beta_{0} \geq \ldots \geq \beta_{n}\right] .
\end{aligned}
$$

Lemma 4. Assume

$$
\begin{aligned}
& (\forall \alpha, \beta)[N F(\alpha, \beta) \Rightarrow \alpha+\beta[n] \leq(\alpha+\beta)[n]] \\
& (\forall m, n)\left[\omega^{m} \cdot(n+1) \leq \omega^{m+1}[n]\right] .
\end{aligned}
$$

Then we have:

(a) $N F(\alpha, \beta) \Rightarrow H_{\alpha}\left(H_{\beta}(n)\right) \leq H_{\alpha+\beta}(n)$.

(b) $\left(H_{\omega^{m}}\right)^{(n+1)}(n+1) \leq H_{\omega^{m+1}}(n)$.

(c) For each primitive recursive function $f$ there exists $m$ such that $(\forall \vec{x})\left[f(\vec{x})<H_{\omega^{m}}(\max \{\vec{x}\})\right]$.

Proof.

(a). From (B5) it follows that $H_{\alpha+\beta}(n)=H_{\alpha+\gamma}(n+1)$ with $\beta[n] \leq \gamma<\beta$. By Lemma 3(a),(b) and I.H. we obtain $H_{\alpha}\left(H_{\beta}(n)\right) \leq H_{\alpha}\left(H_{\gamma}(n+1)\right) \leq H_{\alpha+\gamma}(n+1)$.

(b). From (B6) it follows that $H_{\omega^{m+1}}(n)=H_{\omega^{m} \cdot(n+1)+\gamma}(n+1)$ for some $\gamma<\omega^{m+1}$. By (a) we have $H_{\omega^{m}}^{(n+1)}(n+1) \leq H_{\omega^{m} \cdot(n+1)}(n+1) \leq H_{\omega^{m} \cdot(n+1)+\gamma}(n+1)$.

(c) follows from (a) and (b).

Definition. By PR* we denote the set of all primitive recursive functions which are strictly increasing in each argument. 
Lemma 5. If $N$ satisfies

$$
\begin{aligned}
\left(\exists h \in \mathrm{PR}^{*}\right)\left(\forall n, \alpha_{1}, \ldots, \alpha_{n}\right)\left[\alpha_{1} \geq \ldots\right. & \geq \alpha_{n} \\
\Rightarrow \max \left\{n, N \omega^{\alpha_{1}}, \ldots, N \omega^{\alpha_{n}}\right\} & \leq N\left(\omega^{\alpha_{1}}+\cdots+\omega^{\alpha_{n}}\right) \\
& \left.\leq h\left(\max \left\{n, N \omega^{\alpha_{1}}, \ldots, N \omega^{\alpha_{n}}\right\}\right)\right]
\end{aligned}
$$

(N2) $\quad\left(\exists h \in \mathrm{PR}^{*}\right)(\forall m)\left[m<N \omega^{m} \leq h(m)\right]$,

then there exists a $g \in \mathrm{PR}^{*}$ such that $(\forall \alpha, l)\left[N\left(\omega^{l} \cdot(\alpha+1)\right) \leq g(N \alpha, l)\right]$.

P r o of . Let $\alpha=\omega^{\alpha_{1}}+\cdots+\omega^{\alpha_{k}}+\omega^{m_{1}}+\cdots \omega^{m_{n}}$, where $\alpha_{1} \geq \ldots \geq \alpha_{k} \geq \omega$ and $\omega>m_{1} \geq \ldots \geq m_{n}$. Then $\omega^{l} \cdot(\alpha+1)=\omega^{\alpha_{1}}+\cdots+\omega^{\alpha_{k}}+\omega^{l+m_{1}}+\cdots \cdot \omega^{l+m_{n}}+\omega^{l}$. Thus

$$
\begin{aligned}
& N\left(\omega^{l} \cdot(\alpha+1)\right) \\
& \leq h\left(\max \left\{k+n+1, N \omega^{\alpha_{1}}, \ldots, N \omega^{\alpha_{k}}, N \omega^{l+m_{1}}, \ldots, N \omega^{l+m_{n}}, N \omega^{l}\right\}\right) \\
& \leq h\left(\max \left\{k+n+1, N \omega^{\alpha_{1}}, \ldots, N \omega^{\alpha_{k}}, h\left(l+m_{1}\right), \ldots, h\left(l+m_{n}\right), h(l)\right\}\right) \\
& \leq h(h(l+N \alpha+1)) .
\end{aligned}
$$

\section{Descent functions and Hardy hierarchies}

Definition. By $\mathcal{R}(\tau, N)$ we denote the set of all functions $f: \mathbb{N}^{n} \longrightarrow \mathbb{N}$ with

$$
f(\vec{x})=\min \{k: \delta(\vec{x}, k+1) \nless \delta(\vec{x}, k)\},
$$

where $\delta: \mathbb{N}^{n+1} \longrightarrow \tau$ such that

$(\exists \alpha)\left(\forall \vec{x} \in \mathbb{N}^{n}\right)[\delta(\vec{x}, 0) \leq \alpha]$ and $\left(\exists q \in \mathrm{PR}^{*}\right)(\forall \vec{x}, k)[N \delta(\vec{x}, k) \leq q(\vec{x}, k)]$.

Definition. If $\mathcal{F}$ is a set of functions $f: \mathbb{N} \longrightarrow \mathbb{N}$, then $\operatorname{Cl}(\mathcal{F})$ is the set of all functions $g: \mathbb{N}^{n} \longrightarrow \mathbb{N}$ such that $(\exists f \in \mathcal{F})(\forall \vec{x})[g(\vec{x}) \leq f(\max \{\vec{x}\})]$.

The proof of the following Theorem has been extracted from WaINER [16].

Theor em 1. If $\langle\tau, \cdot[\cdot], N\rangle$ is a normed Bachmann system satisfying (B5), (B6), (N1), (N2), then $\mathcal{R}(\tau, N) \subseteq \mathrm{Cl}\left(\left\{H_{\alpha}: \alpha<\tau\right\}\right)$.

Proof. Let $\delta: \mathbb{N}^{2} \longrightarrow \tau, \alpha<\tau, g \in \mathrm{PR}^{*}$ such that $(\forall n)[\delta(n, 0) \leq \alpha]$ and $(\forall n, k)[N \delta(n, k) \leq g(n, k)]$. (This case can be assumed without loss of generality.) By Lemma 5 we may assume that also $N\left(\omega^{l} \cdot(\xi+1)\right) \leq g(N \xi, l)$ for all $\xi, l$. By Lemma $4(c)$ there is an $m \geq 1$ such that

$$
g(g(n, k+2), l)<H_{\omega^{m}}(\max \{l, n, k\}), \text { for all } l, k, n .
$$

Abbreviations: $\alpha(n, k):=\omega^{m} \cdot \delta(n, k)$ and $f(n, k):=g(g(n, k+1), m)$.

Then we have

$$
\begin{aligned}
N\left(\alpha(n, k+1)+\omega^{m}\right) & =N\left(\omega^{m} \cdot(\delta(n, k+1)+1)\right) \\
& \leq g(N \delta(n, k+1), m) \leq g(g(n, k+1), m)=f(n, k),
\end{aligned}
$$

and

$\left(1^{\prime}\right) \quad \alpha(n, 0) \leq \omega^{m} \cdot \alpha$ and $N \alpha(n, 0) \leq f(n, 0)$,

(2) $f(n, k+1)<H_{\omega^{m}}(\max \{m, n, k\})$. 
From (2) we get

(3) $f(n, k+1)<H_{\omega^{m}}(f(n, k))$.

Now we are going to prove

(4) $\delta(n, k+1)<\delta(n, k) \Rightarrow H_{\alpha(n, k+1)}(f(n, k+1))<H_{\alpha(n, k)}(f(n, k))$.

Indeed: The premise yields $\alpha(n, k+1)+\omega^{m} \leq \alpha(n, k)$ and thus, by (1) and Lemma $3(\mathrm{a}),(\mathrm{d})$,

$$
H_{\alpha(n, k+1)+\omega^{m}}(f(n, k)) \leq H_{\alpha(n, k)}(f(n, k)) .
$$

By (3), Lemma 3(a) and Lemma 4(a) we get

$$
H_{\alpha(n, k+1)}(f(n, k+1))<H_{\alpha(n, k+1)} H_{\omega^{m}}(f(n, k)) \leq H_{\alpha(n, k+1)+\omega^{m}}(f(n, k)) .
$$

This proves (4).

From (4) it follows that

$$
\min \{k: \delta(n, k+1) \nless \delta(n, k)\} \leq H_{\alpha(n, 0)}(f(n, 0)) .
$$

By $\left(1^{\prime}\right),(2)$, Lemma 4(a) we obtain

$$
\begin{aligned}
H_{\alpha(n, 0)}(f(n, 0)) & \leq H_{\omega^{m \cdot \alpha}}(f(n, 0)) \\
& \leq H_{\omega^{m} \cdot(\alpha+1)}(\max \{m, n\}) \\
& \leq H_{\omega^{m} \cdot(\alpha+1)+m}(n) .
\end{aligned}
$$

Le mma 6. If there is an $h \in \mathrm{PR}^{*}$ such that

$$
(\forall \alpha)[N(\alpha+1) \leq h(N \alpha)] \text { and }(\forall \alpha, n)[N(\alpha[n]) \leq h(\max \{N \alpha, n\})],
$$

then $\left\{H_{\alpha}: \alpha<\tau\right\} \subseteq \mathcal{R}(\tau, N)$ and $(\forall n, k)\left[N \delta(n, k) \leq h^{(n+k)}(N \alpha)\right]$.

Proof. By Lemma 3(f) we have $H_{\alpha}(n)=\min \{k: \alpha[n: k+1] \nless \alpha[n: k]\}$ with

$$
\alpha[n: k]:=\alpha+(n-k), \text { for } k \leq n,
$$

and

$$
\alpha[n: k+1]:=(\alpha[n: k])[k], \text { for } k \geq n .
$$

The premises of Lemma 6 yield

$$
(\forall n)[\alpha[n: 0]<\alpha+\omega] \text { and }(\forall n, k)\left[N \alpha[n: k] \leq h^{(n+k)}(N \alpha)\right] .
$$

Hence $H_{\alpha} \in \mathcal{R}(\tau, N)$.

Remark. Theorem 1 and Lemma 6 provide useful criteria for comparing the growth rates of Hardy hierarchies belonging to different normed Bachmann systems. Suppose that $\langle\tau, \cdot[\cdot], N\rangle$ and $\left\langle\tau, \cdot[\cdot]^{\prime}, N^{\prime}\right\rangle$ satisfy the assumptions of Theorem 1 and Lemma 6, respectively, and that there is a function $q \in$ PR $^{*}$ such that $(\forall \alpha<\tau)\left(N \alpha \leq q\left(N^{\prime} \alpha\right)\right)$. Then $\left\{H_{\alpha}^{\prime}: \alpha<\tau\right\} \subseteq \mathrm{Cl}\left(\left\{H_{\alpha}: \alpha<\tau\right\}\right.$ holds for the respective $\mathrm{Hardy}$ hiërarchies.

De f in it io n. Let $\langle\mathcal{A}, \prec\rangle$ be a primitive recursive well-order $(\mathcal{A} \subseteq \mathbb{N}, \prec \subseteq \mathbb{N} \times \mathbb{N})$ of order type $\tau$, and let ord $: \mathbb{N} \longrightarrow \tau$ and $(\cdot)^{*}: \tau \longrightarrow \mathcal{A}$, such that $(\forall \alpha \in \tau)\left[\operatorname{ord}\left(\alpha^{*}\right)=\alpha\right]$ and $(\forall a, b \in \mathcal{A})[b \prec a \Leftrightarrow \operatorname{ord}(b)<\operatorname{ord}(a)]$. Let $\mathcal{R}(\mathcal{A}, \prec)$ be the set of all functions $f: \mathbb{N}^{n} \longrightarrow \mathbb{N}$, with $f(\vec{x})=\min \{k: \Theta(\vec{x}, k+1) \nprec \Theta(\vec{x}, k)\}$, where $\Theta: \mathbb{N}^{n+1} \longrightarrow \mathcal{A}$ is primitive recursive and $(\exists a \in \mathcal{A})(\forall \vec{x})[\Theta(\vec{x}, 0) \preceq a]$. For each set $X$ of functions $f: \mathbb{N}^{n} \longrightarrow \mathbb{N}$ let $\operatorname{PR}[X]$ be the set of all functions which are primitive recursive in $X$. 
Theorem 2. Let $\langle\tau, \cdot[\cdot]\rangle$ be a Bachmann system and assume

(1) $\langle\tau, \cdot[\cdot]\rangle$ satisfies (B5), (B6),

(2) $\left(\exists q \in \mathrm{PR}^{*}\right)(\forall \alpha, \beta)\left[\left(\beta<\alpha \Rightarrow \beta \leq \alpha\left[q\left(\beta^{*}\right)\right]\right]\right.$,

(3) $N:=\lambda \alpha \cdot \alpha^{*}$ satisfies (N1), (N2).

Then $\mathcal{R}(\mathcal{A}, \prec) \subseteq \bigcup_{\alpha<r} P R\left[H_{\alpha}\right]$.

Proof. Let $N^{\prime} \alpha:=q\left(\alpha^{*}\right)$, with $q$ from assumption (2). Obviously $N^{\prime}$ also satisfies (N1), (N2). So $\left\langle\tau, \cdot[\cdot], N^{\prime}\right\rangle$ is a regular Bachmann system satisfying (B5), (B6), (N1), (N2), and therefore, by Theorem $1, \mathcal{R}\left(\tau, N^{\prime}\right) \subseteq \mathrm{Cl}\left(\left\{H_{\alpha}: \alpha<\tau\right\}\right)$.

Now let $f(x)=\min \{k: \Theta(x, k+1) \nprec \Theta(x, k)\}$. We set $\delta(x, k):=\operatorname{ord}(\Theta(x, k))$. Then $f(x)=\min \{k: \delta(x, k+1) \nless \delta(x, k)\}$ and $N^{\prime} \delta(x, k)=q(\Theta(x, k))$. Hence $f \in \mathcal{R}\left(\tau, N^{\prime}\right) \subseteq \operatorname{Cl}\left(\left\{H_{\alpha}: \alpha<\tau\right\}\right)$ and therefore $(\forall x)\left(f(x) \leq H_{\alpha}(x)\right)$ for some $\alpha<\tau$ It follows $f(x)=\min \left\{k \leq H_{\alpha}(x): \Theta(x, k+1) \nprec \Theta(x, k)\right\}$ and thus $f \in \operatorname{PR}\left[H_{\alpha}\right]$.

Theorem 3. Let $\langle\tau, \cdot[\cdot]\rangle$ be a Bachmann system satisfying

$$
(\forall \alpha, \beta, n)[N F(\alpha, \beta) \Rightarrow \alpha+\beta[n]=(\alpha+\beta)[n]]
$$

$(+) \quad\left(\exists q \in \mathrm{PR}^{*}\right)(\forall \alpha)\left[G \alpha:=\min \left\{i: \alpha[0]^{i}=0\right\} \leq q\left(\alpha^{*}\right)\right]$.

Then $\mathcal{R}(\mathcal{A}, \prec) \subseteq \mathcal{R}(\tau, G) \subseteq \bigcup_{\alpha<\tau} \operatorname{PR}\left[H_{\alpha}\right]$.

Proof. By $\left(\mathrm{B}^{\prime}\right)$ we have $(N F(\alpha, \beta) \Rightarrow G(\alpha+\beta)=G \alpha+G \beta)$, and (B6 $\left.6^{\prime}\right)$ yields $G \omega^{m+1}=G \omega^{m}+1$, hence $G \omega^{m}=m+1$. It follows that $G$ satisfies (N1), (N2). Hence, by Lemma $2,\langle\tau, \cdot[\cdot], G\rangle$ is a regular Bachmann system, and from Theorem 1 we obtain $\mathcal{R}(\tau, G) \subseteq \mathrm{Cl}\left\{H_{\alpha}: \alpha<\tau\right\}$.

Now let $f(x)=\min \{k: \Theta(x, k+1) \nprec \Theta(x, k)\}$. We set $\delta(x, k):=\operatorname{ord}(\Theta(x, k))$. Then $f(x)=\min \{k: \delta(x, k+1) \nless \delta(x, k)\}$ and $G \delta(x, k) \leq q\left(\delta(x, k)^{*}\right)=q(\Theta(x, k))$, i. e. $f \in \mathcal{R}(\tau, G)$.

Re m a r $k$. The conditions of the last theorem are satisfied by the standard coding $(\mathcal{E}, \prec \mathcal{E})$ of $\varepsilon_{0}$ and the standard system of fundamental sequences $\cdot[\cdot]_{0}$.

Le mma 7. If $\langle\tau, \cdot[\cdot]\rangle$ is a Bachmann system such that the function $s(a, n):=$ $(\operatorname{ord}(a)[n])^{*}$ is primitive recursive, then $\left\{H_{\alpha}: \alpha<\tau\right\} \subseteq \operatorname{PR}[\mathcal{R}(\mathcal{A}, \prec)]$.

P r o of. Let $\alpha<\tau$ and $a:=\alpha^{*}$. From Lemma 3(f) it follows that

$$
H_{\alpha}(n)=n+\min \{k: \Theta(n, k+1) \nprec \Theta(n, k)\}
$$

with $\Theta(n, 0):=a, \Theta(n, k+1):=s(\Theta(n, k), n+k)$.

\section{Cichon's fundamental sequences and normed Bachmann systems}

In this section we relate (a slightly generalized version of) Cichon's definition $\alpha[n]_{C}:=\max \{\beta<\alpha: N \beta \leq N \alpha+n\}$ to the concept of a normed Bachmann system and its corresponding Hardy hierarchy. Actually in Theorem 4 and Theorem 5 we show that under certain rather weak assumptions both approaches are interchangeable. 
Theorem 4. Let $N: \tau \longrightarrow \mathbb{N}$ be a norm on $\tau$ with

$$
(\forall \alpha)[N 0 \leq N \alpha] \text { and }(\forall \alpha)[N(\alpha+1) \leq N \alpha+1]
$$

and let $p: \tau \longrightarrow \mathbb{N}$ with $(\forall \alpha)[N \alpha \leq p(\alpha)+1 \leq p(\alpha+1)]$. We define $\cdot[\cdot]: \tau \times \mathbb{N} \longrightarrow \tau$ by

$$
0[n]:=0 \text { and } \alpha[n]:=\max \{\beta<\alpha: N \beta \leq p(\alpha+n)\}, \text { for } \alpha>0 .
$$

Let $\left(H_{\alpha}\right)_{\alpha<\tau}$ be the Hardy hierarchy belonging to $\cdot[\cdot]$. Then

(a) $\langle\tau, \cdot[\cdot], N\rangle$ is a normed Bachmann system.

(b) $p(\lambda+n)=N \lambda[n]$, for $\lambda \in$ Lim.

(c) $H_{\alpha}(n)=\max \left\{H_{\beta}(n+1): \beta<\alpha \& N \beta \leq p(\alpha+n)\right\}$, for $\alpha>0$.

Proof.

(b). Let $\lambda \in \operatorname{Lim}$. Then $\lambda[n]<\lambda$ and $N \lambda[n] \leq p(\lambda+n)$, and thus

$$
N \lambda[n] \neq p(\lambda+n) \Rightarrow \lambda[n]+1<\lambda \& N(\lambda[n]+1) \leq p(\lambda+n) .
$$

By maximality of $\lambda[n]$ the latter yields $N \lambda[n]=p(\lambda+n)$.

(a). For $\alpha=\alpha_{0}+1$ we have $\alpha[n]=\max \left\{\beta \leq \alpha_{0}: N \beta \leq p\left(\alpha_{0}+n+1\right)\right\}=\alpha_{0}$, since $N \alpha_{0} \leq p\left(\alpha_{0}+n+1\right)$. Now let $\alpha \in \operatorname{Lim}$. Then

(B1): $N(\alpha[n]+1)=N \alpha[n]+1 \leq p(\alpha+n)+1 \leq p(\alpha+n+1) \Rightarrow \alpha[n]+1 \leq \alpha[n+1]$.

(B3): $\alpha[n]<\beta<\alpha \Rightarrow N \alpha[n]=p(\alpha+n)<N \beta$.

(B4): $N \alpha \leq p(\alpha)+1=N \alpha[0]+1$.

(c). By definition we have $N \alpha[n] \leq p(\alpha+n)$. Hence

$$
H_{\alpha}(n)=H_{\alpha[n]}(n+1) \leq \max \left\{H_{\beta}(n+1): \beta<\alpha \& N \beta \leq p(\alpha+n)\right\}
$$

For the reverse direction we prove by induction on $\alpha$ :

$$
\beta<\alpha \& N \beta \leq p(\alpha+n) \Rightarrow H_{\beta}(n+1) \leq H_{\alpha}(n) .
$$

Indeed: From the premise we get $H_{\alpha}(n)=H_{\alpha[n]}(n+1)$ and $\beta \leq \alpha[n]$. If $\beta=\alpha[n]$ then $H_{\beta}(n+1)=H_{\alpha}(n)$. Now let $\beta<\alpha[n]$. Using $\left(\alpha_{0}+1\right)[n]=\alpha_{0}$ and (b) one easily verifies that $p(\alpha+n) \leq p(\alpha[n]+n+1)$. From $N \beta \leq p(\alpha+n) \leq p(\alpha[n]+n+1)$ by I.H. one obtains $H_{\beta}(n+1)<H_{\beta}(n+2) \leq H_{\alpha[n]}(n+1)=H_{\alpha}(n)$.

Now we show that the roles of $p$ and $\cdot[\cdot]$ in the last theorem can be reversed in some sense.

Theorem 5. Let $\langle\tau, \cdot[\cdot], N\rangle$ be a normed Bachmann system with

$$
(\forall \alpha)[N 0 \leq N \alpha] \text { and }(\forall \alpha)[N(\alpha+1) \leq N \alpha+1] \text {, }
$$

and let $\left(H_{\alpha}\right)_{\alpha<\tau}$ be the corresponding Hardy hierarchy. We define $p: \tau \longrightarrow \mathbb{N}$ by

$$
p(n):=N(n) \text { and } p(\lambda+n):=N \lambda[n], \text { for } \lambda \in \operatorname{Lim} \text {. }
$$

Then the following holds for all $\alpha$ :

(a) $N \alpha \leq p(\alpha)+1 \leq p(\alpha+1)$.

(b) $\alpha[n]=\max \{\beta<\alpha: N \beta \leq p(\alpha+n)\}$, for $\alpha>0$.

(c) $H_{\alpha}(n)=\max \left\{H_{\beta}(n+1): \beta<\alpha \& N \beta \leq p(\alpha+n)\right\}$, for $\alpha>0$. 
Proof.

(a). Let $\alpha=\lambda+n$ with $\lambda \in \operatorname{Lim}$. Then $N \alpha=N \lambda+n \leq N \lambda[0]+n+1 \leq$ $N \lambda[n]+1=p(\alpha)+1 \leq N \lambda[n+1]=p(\alpha+1)$.

(b). If $\alpha=\beta+1$, then $\alpha[n]=\beta, N \beta \leq p(\beta+1) \leq p(\alpha+n)$. If $\alpha \in$ Lim, then $N \alpha[n]=p(\alpha+n)$ and $(\forall \beta<\alpha)(N \beta \leq N \alpha[n] \Rightarrow \beta \leq \alpha[n])$.

(c). This follows from (a), (b) and Theorem 4.

Theorem 6. There exists $N: \tau \longrightarrow \mathbb{N}$ such that $N 0=0, N(\alpha+1)=N \alpha+1$ and $(\forall d \in \mathbb{N})[\{\beta<\alpha: N \beta \leq d\}<\omega]$.

Proof. Let $N^{\prime}: \tau \cap \lim \longrightarrow \mathbb{N}$ be an arbitrary injective function and put $N(\alpha+n):=N^{\prime}(\alpha)+n$ for $\alpha \in$ Lim.

This last theorem yields for example that for every countable ordinal $\tau$ there is an assignment of fundamental sequences which has the Bachmann property (B2). The standard proof of this fact given in RosE [11] is based on a non immediate transfinite recursion!

\section{Applications}

In a first step we concentrate on the ordinal $\varepsilon_{0}:=\min \left\{\xi: \xi=\omega^{\xi}\right\}$, the prooftheoretic ordinal of PA. We assume a standard coding $\langle\mathcal{E}, \prec \mathcal{E}\rangle$ of $\varepsilon_{0}$ in the natural numbers (see, for example, RosE [11] for a definition) such that especially $\mathcal{E}$ and $\prec \mathcal{E}$ are primitive recursive. The standard system of fundamental sequences $\cdot[\cdot]$ is given by the following definition.

$$
\left(\omega^{\alpha} \cdot(\beta+1)\right)[x]:=\omega^{\alpha} \cdot \beta+\omega^{\alpha[x]} \text { if } \alpha \text { is a limit }
$$

and

$$
\left(\omega^{\alpha} \cdot(\beta+1)\right)[x]:=\omega^{\alpha} \cdot \beta+\omega^{\alpha_{0}} \cdot(x+1) \text { if } \alpha=\alpha_{0}+1 .
$$

Let $\left(H_{\alpha}\right)_{\alpha<\tau}$ be the corresponding Hardy hierarchy. One easily verifies that $\left\langle\varepsilon_{0}, \cdot[\cdot]\right\rangle$ is a Bachmann system.

Let $G: \varepsilon_{0} \longrightarrow \mathbb{N}$, where $G(\alpha):=\min \left\{k: \alpha[0]^{k}=0\right\}$. Then $\left\langle\varepsilon_{0}, \cdot[\cdot], G\right\rangle$ is a normed Bachmann system (cf. Lemma 2). Moreover the "axioms" (B5), (B6) are satisfied, and

$$
(\forall \alpha, \beta>0)[\max \{G \alpha, G \beta\}<G(\alpha \# \beta)=G \alpha+G \beta] \text { and }(\forall m)\left[G \omega^{m}=m+1\right] .
$$

Hence by Theorem 1

$$
\mathcal{R}(\tau, G) \subseteq C l\left(\left\{H_{\alpha}: \alpha<\tau\right\}\right)
$$

Now let $N: \varepsilon_{0} \longrightarrow \mathbb{N}$ be a norm with $N 0=0$ and $(\forall \alpha)[N(\alpha+1)=N \alpha+1]$. Let $q \in \mathrm{PR}^{*}$ and $p:=q \circ N$. Let $H_{0}^{N, p}(n):=n$ and

$$
H_{\alpha}^{N, p}(n):=\max \left\{H_{\beta}^{N, p}(n+1): \beta<\alpha \& N \beta \leq p(\alpha+n)\right\}, \text { for } \alpha>0 .
$$

Then by Lemma 6 and Theorem 4 we have

$$
\left\{H_{\alpha}^{N, p}: \alpha<\varepsilon_{0}\right\} \subseteq \mathcal{R}(\tau, N) .
$$

From (1) and (2) we get

$$
\left(\exists q \in \mathrm{PR}^{*}\right)(\forall \alpha)\left[G \alpha \leq q(N \alpha) \Rightarrow\left\{H_{\alpha}^{N, p}: \alpha<\varepsilon_{0}\right\} \subseteq \mathrm{Cl}\left(\left\{H_{\alpha}: \alpha<\tau\right\}\right] .\right.
$$


Now put $p(n):=N(n)$ and $p(\lambda+n):=N(\lambda[n])$. A transfinite induction with the use of Theorem 4 and 5 yields $H_{\alpha}(n)=H_{\alpha}^{N, p}$. So the classical Hardy hierarchy appears as a special case of our approach. We arrive at the following classical result:

Corollary. If the Hardy functions are defined with respect to $\left\langle\varepsilon_{0}, \cdot[\cdot]\right\rangle$, then

$$
\bigcup\left\{\operatorname{PR}\left[\left\{H_{\alpha}\right\}\right]: \alpha<\varepsilon_{0}\right\}=\operatorname{PR}[\mathcal{R}(\mathcal{E}, \prec \varepsilon)] .
$$

Now we look at three concrete examples of norms.

The first norm, $N_{1}$ is given by the depth or rank of the ordinal term when the ordinals less than $\varepsilon_{0}$ are represented by the "constant" 0 and the binary function $\lambda \xi \eta \cdot \omega^{\xi}+\eta$. More precisely, let $N_{1} 0:=0$ and $N_{1} \alpha:=\max \left\{N_{1} \alpha_{1}, N_{1} \alpha_{2}\right\}+1$ if $\alpha=\omega^{\alpha_{1}}+\alpha_{2}>\alpha_{1}, \alpha_{2}$.

The second norm (see CiCHON [5]) is given by the depth of the ordinal term when the ordinals less than $\varepsilon_{0}$ are represented by 0 , and the varyadic function $\lambda \xi_{1} \ldots \xi_{m} \cdot \omega^{\xi_{1}}+\cdots+\omega^{\xi_{m}}$. Let $N_{2} 0:=0$ and $N_{2} \alpha:=\max \left\{1+N_{2} \alpha_{1}, \ldots, 1+N_{2} \alpha_{m}, m\right\}$ if $\alpha=\omega^{\alpha_{1}}+\cdots+\omega^{\alpha_{m}}>\alpha_{1} \geq \ldots \geq \alpha_{m}$.

Finally we consider a norm which is given by the number of symbols (except 0 and + ) which occur in an ordinal term (see WEIERMANN [19]). Let $N_{3}(0):=0$ and $N_{3} \alpha:=1+N_{3} \alpha_{1}+\cdots+1+N_{3} \alpha_{m}$ if $\alpha=\omega^{\alpha_{1}}+\cdots+\omega^{\alpha_{m}}>\alpha_{1} \geq \ldots \geq \alpha_{m}$. (It can be seen easily that $N_{3}$ equals the function $G$ from the discussion above.)

Let $h(x):=3^{x+1}, p_{i}:=h \circ N_{i}$ and $\alpha[n]_{i}:=\max \left\{\beta<\alpha: N_{i} \beta \leq p_{i}(\alpha+n)\right\}$. Then for $i=1,2,3$ the "axioms" (N1) $i$ and (N2) 2 are satisfied for $h$ and $N_{i}$. Moreover the axioms (B5) $i$ and (B6) $)_{i}$ are satisfied for $\cdot[]_{i}$ and $i \in\{1,2,3\}$. Therefore, $\left\{H_{\alpha}^{N_{i}, p_{i}}: \alpha<\varepsilon_{0}\right\} \subseteq \mathcal{R}\left(\varepsilon_{0}, N_{i}\right) \subseteq \operatorname{Cl}\left(\left\{H_{\alpha}^{N_{i}, p_{i}}: \alpha<\varepsilon_{0}\right\}\right)$. Furthermore, the systems $\left\langle\varepsilon_{0}, \cdot[\cdot]_{i}, N_{i}\right\rangle$ are normed Bachmann systems. (In WEIERMANN [19] it is shown that every provably total function of PA is bounded by $H_{\alpha}^{N_{3}, p_{3}}$ for some $\alpha<\varepsilon_{0}$.)

Corollary. If $i \in\{1,2,3\}$, then

$$
\bigcup\left\{\operatorname{PR}\left[\left\{H_{\alpha}^{N_{i}, p_{i}}\right\}\right]: \alpha<\varepsilon_{0}\right\}=\operatorname{PR}[\mathcal{R}(\mathcal{E}, \prec \mathcal{E})] .
$$

Now we turn to a strong generalization of this example.

Let $\mathcal{T}(M)$ and $<\subseteq \mathcal{T}(M) \times \mathcal{T}(M)$ be defined as in RATHJEN [10]. This ordinal notation system is characteristic for the theory KPM formalizing a recursively Mahlo universe. Let $\langle\mathcal{M}, \prec \mathcal{M}\rangle$ be a standard coding of $\langle\mathcal{T}(M),<\rangle$ in the natural numbers. Define $N: \mathcal{T}(M) \longrightarrow \omega$ as follows:

1. $N 0$,

2. $N M:=1$,

3. $N \oplus\left(\alpha_{1}, \ldots, \alpha_{n}\right):=N \alpha_{1}+\cdots+N \alpha_{n}$,

4. $N \bar{\omega}^{\alpha}:=N \alpha+1$,

5. $N \bar{\varphi} \alpha \beta:=1+N \alpha+N \beta$,

6. $N \psi \kappa \alpha:=1+N \kappa+N \alpha$,

7. $N Z \alpha:=N \alpha+1$.

For $\alpha \in \mathcal{T}(M)$ let $\alpha[x]_{M}:=\max \left\{\beta<\alpha: N \beta \leq 3^{3^{N \alpha+x+1}}\right\}$. Then we get the following corollary. 
Corollary. If the Hardy functions are defined with respect to $\left\langle\mathcal{T}(M), \cdot[\cdot]_{M}\right\rangle$, then

$$
\bigcup\left\{\operatorname{PR}\left[\left\{H_{\alpha}\right\}\right]: \alpha \in \mathcal{T}(M)\right\}=\operatorname{PR}[\mathcal{R}(\mathcal{M}, \prec \mathcal{M})] .
$$

Furthermore, for every function $f: \omega \longrightarrow \omega$ which is provably total in KPM there is an $\alpha \in \mathcal{T}(M)$ such that $f(x)<H_{\alpha}(x)$ for all $x<\omega$.

We think that our method also applies to arbitrary so-called natural well-orderings. But since so far a precise definition of this notion has not been given we can only give the construction of fundamental sequences for notation systems which satisfy certain not too restrictive "naturalness"-assumptions. (These assumptions are satisfied by all notation systems used in proof-theory so far.) We assume that we are given an inductively defined primitive recursive set of terms $T$, where the underlying set of function symbols is finite, together with a primitive recursive well-ordering $<_{T}$ on $T$. We assume furthermore, that there is a zero-constant $0^{*}$ in $T$ which denotes the $<_{T}$-minimal element of $T$ and that (among the function symbols in question) there is a binary function $+^{*}$ (respectively varyadic function $+^{*}$ such that the order type of $t_{1}+{ }^{*} \cdots+^{*} t_{n}$ with respect to $<T$ is the sum of the order types of $t_{1}, \ldots, t_{n}$ with respect to $<_{T}$. Then we define a norm $N$ of an ordinal term $t$ by taking the number of occurrences of the "on+"-function symbols and nonzero-constants which occur in $T$. As an assumption on $T$ we additionally demand that $N 1^{*}=1$, where $1^{*}$ denotes the $<_{T}$-successor of $0^{*}$. Then $N$ satisfies the assumptions of Theorem 4 , and therefore by using Cichon's method for defining assignments of fundamental sequences we automatically obtain a normed Bachmann system $\langle\tau, \cdot[\cdot], N\rangle$.

As shown in Theorems 4 and 5 the theory of Hardy hierarchies could as well be developed without any reference to fundamental sequences (using instead the auxiliary functions $p$ ). For the reader's convenience we present one of our main results (namely the comparison between descent functions and the Hardy hierarchy) in a formulation which does not refer to fundamental sequences at all.

Theorem. Let $N: \tau \longrightarrow \mathbb{N}$ be a norm on $\tau$ with

(1) $(\forall \boldsymbol{\alpha}>0)[N \alpha>N 0]$,

(2) $N \omega^{m}=m+1$,

(3) $\alpha, \beta>0 \Rightarrow N(\alpha \# \beta)=N \alpha+N \beta$.

Let $p: \tau \longrightarrow \mathbb{N}$ such that

(4) $p(\alpha)<p(\alpha+1)$,

(5) $N \alpha \leq p(\alpha)+1 \leq h(N \alpha)$, for some $h \in \mathrm{PR}^{*}$,

(6) $N\left(\omega^{m} \cdot n\right) \leq p\left(\omega^{m+1}+n\right)$,

(7) $N F(\alpha, \beta) \Rightarrow N \alpha+p(\beta) \leq p(\alpha+\beta)$.

Let $H_{0}^{N, p}(n):=n$ and $H_{\alpha}^{N, p}(n):=\max \left\{H_{\beta}^{N, p}(n+1): \beta<\alpha \& N \beta \leq p(\alpha+n)\right\}$, for $\alpha>0$. Then $\mathrm{Cl}(\mathcal{R}(\tau, N))=\mathrm{Cl}\left(\left\{H_{\alpha}^{N, p}: \alpha<\tau\right\}\right)$.

Proof. Let

$$
0[n]:=0 \text { and } \alpha[n]:=\max \{\beta<\alpha: N \beta \leq p(\alpha+n)\} \text {, for } \alpha>0 .
$$


By Theorem $4\langle\tau, \cdot[\cdot], N\rangle$ is a normed Bachmann system and $\left(H_{\alpha}^{N, p}\right)_{\alpha<\tau}$ is the corresponding Hardy hierarchy. By definition and (2), (3), (5) we have $N \alpha[n] \leq p(\alpha+n) \leq$ $h(N \alpha+n)$. Therefore, Lemma 6 yields $\left\{H_{\alpha}: \alpha<\tau\right\} \subseteq \mathcal{R}(\tau, N)$. According to Theorem 1 it remains to verify (B5), (B6), (N1), (N2).

(N1) follows from (3) and (1).

(N2) follows from (2).

(B6) follows from (6).

(B5) follows from (7). $[N(\alpha+\beta[n])=N \alpha+N(\beta[n]) \leq N \alpha+p(\beta+n) \leq p(\alpha+\beta+n)$. $]$

Remark . (4) - (7) are satisfied if for example $p(\alpha):=f\left(2^{N \alpha}\right)$ with $f \in \mathrm{PR}^{*}$. ad (6): $N\left(\omega^{m} \cdot n\right)=\left(N \omega^{m}\right) \cdot n<2^{N \omega^{m+1}+n} \leq f\left(2^{N\left(\omega^{m+1}+n\right)}\right)=p\left(\omega^{m+1}+n\right)$. ad (7): $N \alpha+p(\beta)=N \alpha+f\left(2^{N \beta}\right) \leq f\left(2^{N \alpha+N \beta}\right)=p(\alpha+\beta)$.

Finally assume additionally that the norm $N$ is primitive recursive and that the computation of $\max \left\{s<_{T} t: N s \leq p\left(t+{ }^{*} n\right)\right\}$ (with respect to $<_{T}$ ) can be done primitive recursively in the arguments $t$ and $n$. (These assumptions are also satisfied by all notation systems used in proof-theory so far.) Then the system $\left\langle\left\langle T,\left\langle_{T}\right\rangle_{,} \cdot[\cdot], N\right\rangle\right.$ is p.r.-regulated in the sense of ZEMKE [22]. Therefore, as an immediate corollary of the main theorem of [22] we obtain the hierarchy equivalence property for these ordinal notation systems!

\section{References}

[1] Buchrolz, W., and S. S. WaINER, Provable computable functions and the fast growing hierarchy. In: Contemporary Mathematics 65 (1987), American Mathematical Society, pp. $179-198$.

[2] Buchrolz, W., An independence result for $\left(\Pi_{1}^{1}-C A\right)+B I$. Annals Pure Appl. Logic 33 (1987), 131 - 155.

[3] Cichon, A., A short proof of two recently discovered independence results using recursion theoretic methods. Proc. Amer. Math. Soc. (4) 87, $704-706$.

[4] CichoN, A., Bounds for derivation lengths from termination proofs. Technical report CSD-TR-622 (unpublished), Department of Computer Science, Surrey, London, England, 1990.

[5] Crchon, A., Termination orderings and complexity characterizations. In: Proof Theory (P. Aczel et al., eds.), Cambridge University Press 1992, pp. $171-193$.

[6] Dennis-Jones, E. C., and S. S. Wainer, Subrecursive hierarchies via direct limits. In: Computation and Proof Theory. Lecture Notes in Mathematics 1104, Springer-Verlag, Berlin-Heidelberg- New York 1984, pp. 118 - 128.

[7] Friedman, H., and M. Sheard, Elementary descent recursion and proof theory. Manuscript 1993, unpublished.

[8] Kadota, N., and K. Aoyama, Some extensions of built-upness on systems of fundamental sequences. Zeitschrift Math. Logik Grundlagen Math. 36 (1990), 357 - 364.

[9] KreISEL, G., On the interpretation of non-finitist proofs II. J. Symbolic Logic 17 (1952), $43-58$.

[10] Rathjen, M., Proof-theoretic analysis of KPM. Archive Math. Logic 30 (1991), $377-403$.

[11] Rose, H. F., Subrecursion: Functions and Hierarchies. Oxford University Press 1984. 
[12] Schmid, D., Built-up systems of fundamental sequences and hierarchies of numbertheoretic functions. Archiv Math. Logik Grundlagenforsch. 18 (1976), 47 - 53. Postscript ibid. 145 - 146.

[13] SMITH, R., The consistency strength of some finite forms of the Higman and Kruskal theorems. In: Harvey Friedman's Research on the Foundations of Mathematics (L. A. Harrington et al., eds.), North-Holland Publ. Comp., Amsterdam 1985, pp. $119-136$.

[14] Schwichtenberg, H., Eine Klassifikation der $\varepsilon_{0}$-rekursiven Funktionen. Zeitschrift Math. Logik Grundlagen Math. 17 (1971), 61 - 74.

[15] Takeuti, G., Proof Theory. Second edition. North-Holland Publ. Comp., Amsterdam 1987.

[16] WAINER, S. S., A classification of the ordinal recursive functions. Archiv Math. Logik Grundlagenforsch. 13 (1970), 136 - 53.

[17] WAINER, S. S., Ordinal recursion and a refinement of the ordinal recursive functions. J. Symbolic Logic 37 (1972), 281 - 292.

[18] WAINER, S. S., Computability - Logical and recursive complexity. In: Logic, Algebra and Computation (F. L. BAUER, ed.), Springer ASI Series F, Volume 79, SpringerVerlag, Berlin-Heidelberg-New York, pp. 237 - 264.

[19] WeIERMANN, A., A simple characterization of the provably recursive functions of PA, $\mathrm{PA}+T I(\prec)$ and $K \mathrm{~K} \omega$. Submitted 1993.

[20] WeIERMaNN, A., Investigations on slow versus fast growing (for large countable ordinals). Münster 1993 (unpublished).

[21] WeiermanN, A., Bounding derivation lengths with slow growing functions. Münster 1993 (unpublished).

[22] ZEMKE, F., P.r.-regulated systems of notation and the subrecursive hierarchy equivalence property. Trans. Amer. Math. Soc. 234 (1977), 89 - 118.

(Received: August 20, 1993) 